\section{Usefulness of the Basophil Activation Test to Confirm Beer Allergy}

Entrala $\mathrm{A}^{1}$, Dominguez-Ortega $\mathrm{J}^{1,3,4}$, Gonzalez-Muñoz $\mathrm{M}^{2}$, Fiandor $\mathrm{A}^{1,3}$, Quirce $\mathrm{S}^{1,3,4}$

${ }^{1}$ Department of Allergy, Hospital La Paz, Madrid, Spain ${ }^{2}$ Department of Immunology, Hospital La Paz, Madrid, Spain

${ }^{3}$ Hospital La Paz Institute for Health Research (IdiPAZ), Madrid, Spain

${ }^{4}$ CIBER de Enfermedades Respiratorias, Ciberes, Madrid, Spain

J Investig Allergol Clin Immunol 2018; Vol. 28(4): 279-280

doi: 10.18176/jiaci.0266

Key words: Basophil activation test. Beer allergy. Beer-induced anaphylaxis. Barley. Cofactors.

Palabras clave: Test de activación de basófilos. Alergia a la cerveza. Anafilaxia por cerveza. Cebada. Cofactores.

Beer is one of the world's most widely consumed beverages. It is produced by fermentation of malted barley, to which brewer's yeast and hops (Humulus lupulus) are added for flavoring [1]. The main types of yeast used in the fermentation process come from the genus Saccharomyces, often Saccharomyces cerevisae or Saccharomyces carlsbergensis. Allergic reactions to beer are rare [2,3]. Moreover, worldwide, sensitization patterns differ depending on local customs and beer ingredients [4]. In Europe, most cases of beer allergy reported to date have been associated with hypersensitivity to barley and malt, for which a major band at $38 \mathrm{kDa}$ has been implicated as the main culprit allergen [5], as has nonspecific lipid transfer protein (LTP) $[1,6]$. Furthermore, in a sensitized individual, the presence of enhancing factors such as alcohol, nonsteroidal anti-inflammatory drugs (NSAIDs), and exercise can explain why some reactions lead to anaphylaxis, while in other cases, the allergen elicits a milder reaction or is even tolerated. Hence, ascertaining the effect of cofactors is critical if we are to prevent the development of a severe anaphylactic reaction. The basophil activation test (BAT) has become a reliable diagnostic tool in the evaluation of food allergy $[7,8]$ and can be used before oral challenge testing, especially if a cofactor such as NSAIDs is suspected of being implicated in the reaction [9]. We present a case of beer-induced anaphylaxis confirmed with a positive BAT result to barley and beer, with a negative effect of dexketoprofen (NSAID) as a cofactor. A 43-year-old woman with no significant previous medical history presented at the emergency department with edema (tongue, uvula, and lips), nasal congestion, cutaneous erythema, and generalized pruritic wheals, which appeared immediately after intake of 1664 Kronenbourg beer, potato chips, and anchovy-stuffed olives. Symptoms resolved after treatment with antihistamines and systemic corticosteroids. The patient had taken a tablet of dexketoprofen (Enantyum, Menarini) 5 hours before the episode. She complained of previous digestive discomfort when drinking beer and later tolerated the other foods involved in the reaction. The results of skin prick tests (SPTs) for rye flour, barley, gliadin, Anisakis, and Saccharomyces were all positive. SPTs were also positive for 5 brands of beers (Kronenbourg, Indian Pale, and Mahou, which only contain barley; Corona, with barley and corn; and Erdinger, with barley and wheat). Total serum $\operatorname{IgE}$ was $89.10 \mathrm{kU} / \mathrm{L}$. Specific IgE was positive for barley $(1.27 \mathrm{kU} / \mathrm{L})$, but negative for grass pollen, Aspergillus fumigatus, Alternaria alternata, Cladosporium herbarum, Mucor racemosus, Penicillium notatum, Humulus lupulus, wheat, rye, gluten, rPru p 3 (LTP), rTri a 14 (LTP), yeast (Saccharomyces cerevisae), rTri a 19 ( $\omega$-5-gliadin). The basal tryptase level was $4.85 \mu \mathrm{g} / \mathrm{L}$. The patient tolerated oral challenge with dexketoprofen but refused to undergo oral challenge with beer or barley. She was advised to avoid ingestion of beers and barley.

The BAT was performed by incubating $100 \mu$ Lof heparinized blood with dexketoprofen $(1 \mathrm{mg} / \mathrm{mL}-0.1 \mathrm{mg} / \mathrm{mL})$, commercial beers $(1 / 5$ and $1 / 50)$, and barley flour $(1 / 10-1 / 10000$ prick test extract, LABORATORIOS LETI, S.L., $1250 \mu \mathrm{g}$ protein/mL in $2 \mathrm{~mL}$ ). Negative and positive controls were included by incubating blood without allergens and $20 \mu \mathrm{L}(10 \mathrm{mg} / \mathrm{mL})$ of anti-IgE (Ingenasa), respectively. Cells were stained with a mixture containing anti-CD63-FITC, anti-CD123-PE, and anti-HLADR-PerCP-conjugated antibodies (BD Bioscience). Basophil activation was determined by $\mathrm{CD} 63$ upregulation in a FACSCalibur flow cytometer (BD Bioscience). The result was considered positive when the percentage of basophils activated after stimulation with the allergens was $>5 \%$ and the stimulation index (the ratio between the percentage of activated basophils after stimulation and percentage of activated basophils in the negative control) was $>2$. In order to assess the effect of dexketoprofen on basophil activation by barley flour, we followed an experimental approach described elsewhere [9]. Whole blood was pre-incubated with the drug at $3 \mu \mathrm{g} / \mathrm{mL}$ (therapeutic plasma concentration [10]) for 10 minutes and then with barley flour extract at suboptimal concentrations (1/1000 and 1/10 000), although maximum basophil activation was not achieved. The patient showed $2 \%$ and $65 \%$ of activated basophils in the negative and positive assays, respectively. The BAT was negative for dexketoprofen $(2 \%)$ but positive for barley flour (63\%), Mahou (63\%), Erdinger (66\%), and Corona (58\%). No cofactor effect was

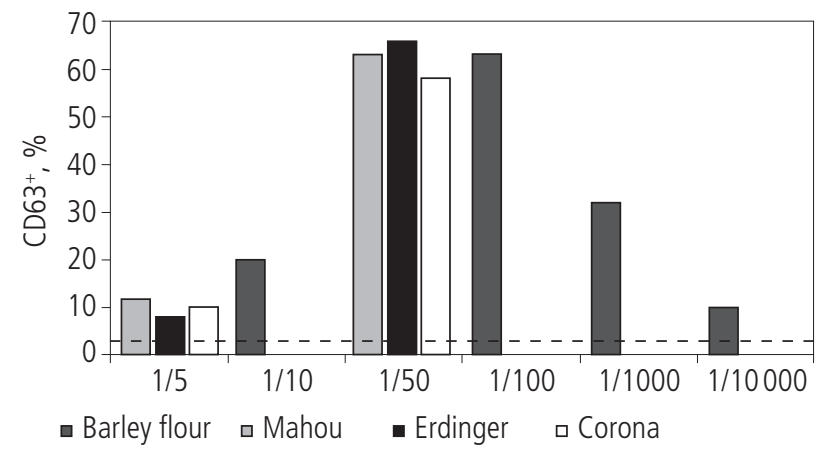

Figure. CD63 upregulation induced by commercial beers and barley flour. Bars represent the percentage of $\mathrm{CD}^{+} 3^{+}$basophils after challenge with serial dilutions of allergens. The dotted line marks $5 \%$ activated basophils. 
documented, thus confirming beer as the only agent involved in the reaction (Figure). BAT was also performed in 3 healthy controls, with no activation observed $(<4 \%)$. To the best of our knowledge, this is the first reported positive BAT result for beer allergy. Although the gold standard for diagnosis of food allergy is controlled oral challenge, BAT could play a role in beer allergy, especially when drugs are involved in the reaction or when a challenge test is not indicated.

\section{Acknowledgments}

To Isabel Coman for correcting the text and Rocio Heredia for technical work.

\section{Funding}

The authors declare that no funding was received for the present study.

\section{Conflicts of Interest}

The authors declare that they have no conflicts of interest.

\section{References}

1. Quercia O, Zoccatelli G, Stefanini GF, Mistrello G, Amato S, Bolla $M$, et al. Allergy to beer in LTP-sensitized patients: beers are not all the same. Allergy. 2012;67:1186-9.

2. Inoue $T$, Yagami $A$, Shimojo $N$, Hara $K$, Nakamura $M$, Matsunaga K. Case of immediate hypersensitivity to beer. J Dermatol. 2016;43:690-2.

3. Herzinger T, Kick G, Ludolph-Hauser D, Przybilla B. Anaphylaxis to wheat beer. Ann Allergy Asthma Immunol. 2004;92:673-5.

4. Song Z, Chen W, Huang X, Zhou X, Luo J, Wang H, et al. Sensitization to beer ingredients with beer allergy: a clinical study of 20 cases. Int Arch Allergy Immunol. 2014;163:13541.

5. Figueredo E, Quirce S, Del Amo A, Cuesta J, Arrieta I, Lahoz $C$, et al. Beer-induced anaphylaxis: identification of allergens. Allergy. 1999;54:630-4.

6. Asero R, Mistrello G, Roncarolo D, Amato S, Van Ree R. A case of allergy to beer showing cross-reactivity between lipid transfer proteins. Ann Allergy Asthma Immunol. 2001;87:65-7

7. Hoffmann HJ, Santos AF, Mayorga C, Nopp A, Eberlein B, Ferrer $M$, et al. The clinical utility of basophil activation testing in diagnosis and monitoring of allergic disease. Allergy. 2015;70:1393-1405.

8. Kang M, Song W, Park H, Lim K, Kim S, Lee S, et al. Basophil Activation Test with Food Additives in Chronic Urticaria Patients. Clin Nutr Res. 2014;3:9-16.

9. Pascal M, Muñoz-Cano R, Milà J, Sanz ML, Diaz-Perales A, Sánchez-López J, et al. Nonsteroidal anti-inflammatory drugs enhance lgE-mediated activation of human basophils in patients with food anaphylaxis dependent on and independent of nonsteroidal anti-inflammatory drugs. Clin Exp Allergy. 2016;46:1111-9.
10. Schulz M, Iwersen-Bergmann $S$, Andresen $H$, Schmoldt A. Therapeutic and toxic blood concentrations of nearly 1,000 drugs and other xenobiotics. Crit Care. 2012;16:136.

Manuscript received January 4, 2018; accepted for publication April 11, 2018

Ana Entrala Bueso

Allergy Department Hospital Universitario La Paz Paseo de la Castellana, 261 28046 Madrid, Spain E-mail: anaentrala@gmail.com 